\title{
Experimental and Numerical Investigation of Station Keeping Control for Ultra-Silent Autonomous Underwater Vehicle
}

Yuhao Zeng ( $M$ M201871635@hust.edu.cn )

Huazhong University of Science and Technology

Huan Zheng

Wuhan Marine Communication Institute

Zu-Yao Yu

Huazhong University of Science and Technology

Original Article

Keywords: Station keeping, Autonomous underwater vehicle, Sliding mode control

Posted Date: July 2nd, 2020

DOI: https://doi.org/10.21203/rs.3.rs-39250/v1

License: (c) (i) This work is licensed under a Creative Commons Attribution 4.0 International License.

Read Full License 


\section{Title page}

\section{Experimental and Numerical Investigation of Station Keeping Control for Ultra-Silent Autonomous Underwater Vehicle}

Yu-Hao Zeng, born in 1996, is currently a master candidate at School of Naval Architecture and Ocean Engineering, Huazhong University of Science and Technology, China. His research interests include underwater vehicle modelling and control, hydraulic system design. Tel: +86-13407195907; E-mail: M201871635@hust.edu.cn

Huan Zheng, born in 1988, is currently an engineer at Wuhan Marine Communication Institute, China.

Zu-Yao Yu, born in 1972, is currently a professor at Huazhong University of Science and Technology, China.

Corresponding author: Zu-Yao Yu E-mail: M201871635@hust.edu.cn 


\title{
Experimental and Numerical Investigation of Station Keeping Control for Ultra- Silent Autonomous Underwater Vehicle
}

\author{
Yu-Hao Zeng ${ }^{1} \cdot$ Huan Zheng ${ }^{2}$ Zu-Yao Yu ${ }^{1}$
}

Received June xx, 202x; revised February xx, 202x; accepted March xx, 202x

(C) Chinese Mechanical Engineering Society and Springer-Verlag Berlin Heidelberg 2017

\begin{abstract}
In this paper, we consider station keeping control law for ultra-silent autonomous underwater vehicle(ULSI-AUV) based on the sliding mode controller. The mechanical system of ULSI-AUV prototype is designed and manufactured, and the electrical system is selected and installed. The kinematic and dynamics model is established. The goal of station keeping control is to make the ULSI-AUV prototype fixed-depth and anti-rolling to carry out underwater operations. Numerical results show the effectiveness of this method. In addition, real-time experimental results also prove the effectiveness of the proposed algorithm.
\end{abstract}

Keywords: Station keeping - Autonomous underwater vehicle • Sliding mode control

\section{Introduction}

Nowadays, people's activities on the marine, river and other underwater environments are becoming more and more widespread $^{[1-2]}$. This puts forward certain requirements for underwater communications. At present, most underwater equipment adopts acoustic communication. Although acoustic communication has been applied well, there are still some inherent shortcomings, such as narrow bandwidth, high delay, etc. ${ }^{[3-5]}$ Therefore, underwater antennas experiments, also known as radiofrequency(RF) communication technological experiments, have also become one of the

\footnotetext{
$\triangle \quad \mathrm{Zu}-\mathrm{Yao} \mathrm{Yu}$ M201871635@hust.edu.cn

1. Huazhong University of Science and Technology, Wuhan 430070, China

2. Wuhan Marine Communication Institute, 430070, China
}

indispensable parts in ocean research in recent years. ${ }^{[6-7]}$

The ultra-silent autonomous underwater vehicle (ULSIAUV) which used to carry the underwater antennas is the key part of the experiment. The controllability of AUV plays a key role during this experiment because the depth and attitude of the underwater antenna must be limited within a small range, which needs the dynamic station keeping control for the AUV.

Although a great deal of research has been done on the control law of AUV around the world, compared with path following and trajectory tracking, there are still limited attempts in station keeping control. J. H. Li has designed a sort of AUV platform to overcome strong currents and keep station ${ }^{[8]}$. Mohan S. has proposed a power efficient dynamic station keeping control law for an elongated shape AUV ${ }^{[9]}$. Koh has adopted a special configuration of thrusters and uses a PD controller to solve the station keeping problem of a remotely operated vehicle (ROV) ${ }^{[10]}$. A kind of robust station keeping controller has been proposed by Serrani under velocity measurement offset ${ }^{[11]}$. And other available kinds of literature have focused on the unknown disturbance or wave currents ${ }^{[12-13]}$.

However, the ULSI-AUV only allows weak current to pass through the thruster, i.e. the propeller must always keep idling, to avoid the electromagnetic interference to the antenna. In this case, the thruster arrangement in the above documents is not very reliable.

To solve the problem above, the vehicle is designed as shown in Figure 1. Refer to some submersible unmanned aerial vehicle ${ }^{[14-15]}$ and special-shaped underwater vehicle $^{[16-20]}$, ULSI-AUV adopts underactuated structure, and four vertical thrusters of AUV are symmetrically installed on $x y$-plane, and each thruster has the same thrust 
dynamic characteristics in both forward and backward directions. This kind of thrust mechanism can be used to decouple the control force, therefore keep the depth and anti-rolling conveniently. Moreover, the geometric arrangement of thrusters is relatively dispersed, and the electrical connection mode is a parallel connection, thus greatly reducing the interference of internal current to RF experiments.

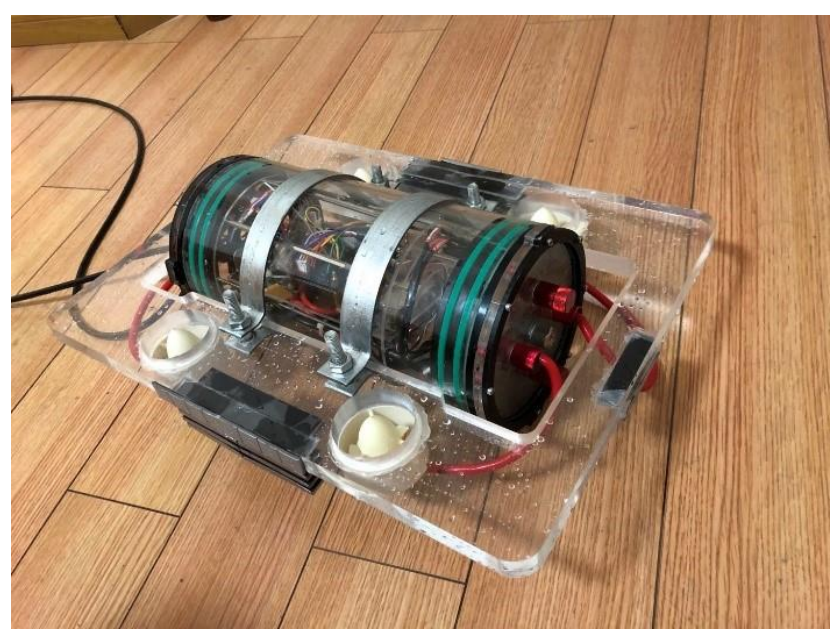

Figure 1 The ULSI-AUV Prototype

The work introduced in this paper describes a scaleddown AUV prototype and realizes the research, development and design of the control strategy, so as to make appropriate adjustments in the actual AUV carrying underwater antenna in the future. Here, we focus on three aspects: the mechanical and electronic system design of the prototype, the derivation of the mathematical model describing the kinematics and dynamics of the system, and the realization of the dynamic station keeping control law in the engineering basin experiment.

The remainder of this paper is organized as follows. Section 2 introduces the mechanical and electronic system design of the ULSI-AUV. Section 3 presents the mathematical model of ULSI-AUV and some differences with conven-tional AUV. In Section 4, we design a controller for ULSI-AUV based on sliding mode and prove its convergence based on Lyapunov theory and Barbalat lemma. The numerical and experimental results are presented in Section 5. Conclusions are drawn in Section 6.

\section{System Design of ULSI-AUV}

The ULSI-AUV prototype comprises a mechanical system and an electrical system. The mechanical system composition of the ULSI-AUV prototype can be seen from Figure 2, it comprises a watertight enclosure, two end covers, two flanges, a set of threading screws, a set of snap rings, two frames and some counterbalance components. The watertight enclosure and the external frame are fixed together by four semicircular snap rings. The thrusters and the counterbalance components are fixed on the bottom of the external frame to lower the centre of gravity and enhance self-stability, while the circuit board and other electronic components are fixed on the internal frame. The sealing groove on the flange is used for placing the $\mathrm{O}$-shape sealing ring to realize axial/radial multiple sealing. A set of holes are machined on the end cover, and threading screws on the holes connect the internal circuit and the external thrusters. Threading screws are also sealed with the end cap through O-ring seals, while self-sealing is a way of encapsulating with epoxy resin.

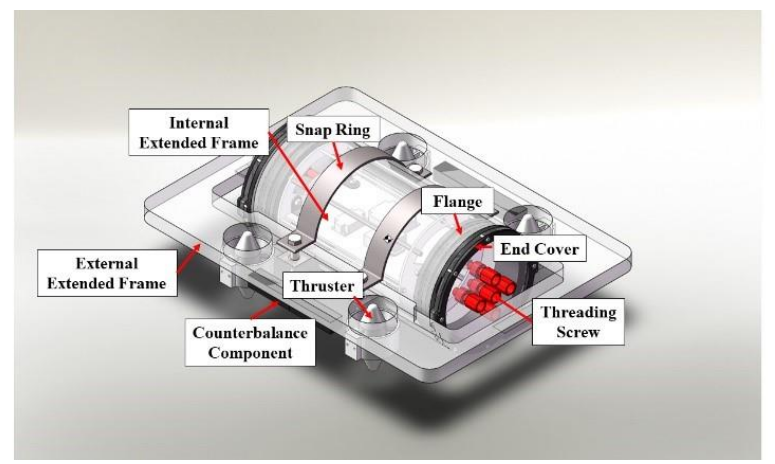

Figure 2 The ULSI-AUV 3D Model

The schematic diagram of the electrical system is shown in Figure 3, it comprises a control subsystem, a communication subsystem and the power supply. The control circuit adopts STM32F1 series micro-controller (MCU) as the central processing unit. The depth and attitude information collected by the depth sensor and the attitude and heading reference system (AHRS), which is used as the digital input of the control system. The PWM wave generated by the controller is the digital output to the bidirectional brushless electronic tunings, which is directly connected to the brushless direct current (BLDC) thrusters, thus changing the thrust, adjusting the attitude and depth, and achieving dynamic positioning balance.

The ULSI-AUV prototype communicates with the host computer in real-time through cable communication. The depth, attitude and PWM value can be transmitted to the engineering software of the host computer in real-time.

Finally, the ULSI-AUV prototype uses a DC regulated power supply placed on the ground to supply power through the cables. More details such as communication protocol are also indicated in Figure 3. 


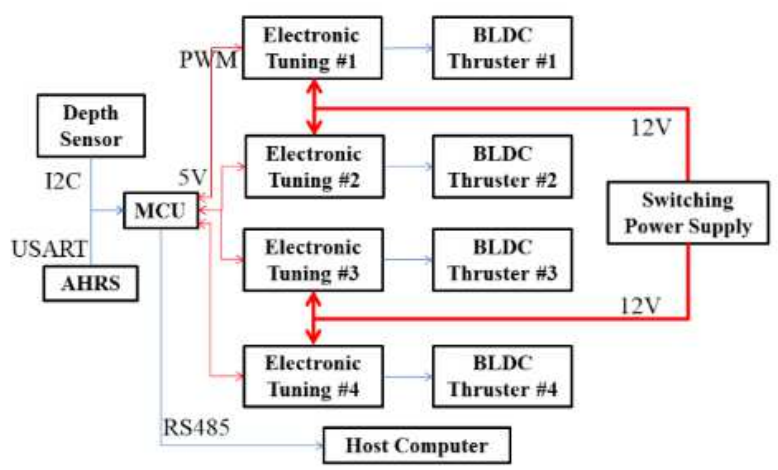

Figure 3 The ULSI-AUV's Electrical System

This prototype adopts low-cost mechanical and electronic components to facilitate the replacement and maintenance of experimental equipment. Components with higher performance and better reliability will be used in future experiments. Table 1 shows the overall design parameters and specification. It should be noted that all hydro-dynamic parameters are obtained with simulation from CFD, and the remaining parameters are also based on measurements of ULSI-AUV prototype.

Table 1 Main design parameters

\begin{tabular}{cl}
\hline Parameters & \multicolumn{1}{c}{ Value } \\
\hline Mass & $m=7.68 \mathrm{~kg}$ \\
Centre of gravity & $r_{B}=[-0.03,0.60,-5.56]^{T} \mathrm{~m}$ \\
Centre of buoyancy & $r_{G}=[0,0,0]^{T} \mathrm{~m}$ \\
Inertia tensor & $I=\operatorname{diag}(0.12,0.08,0.19) \mathrm{kg} \cdot \mathrm{m}^{2}$ \\
Position of four thrusters & $r_{T}=[ \pm 0.11, \mp 0.13,0.03]^{T} \mathrm{~m}$ \\
Crucial hydrodynamics & $K_{p|p|}=5.27 \mathrm{~N} \cdot \mathrm{m} /(\mathrm{rad} / \mathrm{s})^{2}$ \\
coefficients & $M_{q|q|}=7.66 \mathrm{~N} \cdot \mathrm{m} /(\mathrm{rad} / \mathrm{s})^{2}$ \\
& $Z_{w|w|}=78.74 \mathrm{~m} /(\mathrm{rad} / \mathrm{s})^{2}$ \\
Design velocity & $v_{\text {down }}=0.06 \mathrm{~m} / \mathrm{s}$ \\
& $v_{u p}=-0.1 \mathrm{~m} / \mathrm{s}$ \\
\hline
\end{tabular}

\section{Mathematical Modelling}

A general mathematical model of AUV consists of kinematics model and dynamics model ${ }^{[21]}$. The kinematics model, which describes the geometrical relationship between inertia-fixed reference frame(I-FRAME) and the body-fixed reference frame(B-FRAME) motions, can be written as

$$
\dot{\xi}=J(\Theta) v
$$

The dynamics model, which mainly describes the relationship between the speed vector of the AUV and the force and moment, can be given by

$$
M \dot{\boldsymbol{v}}+C(\boldsymbol{v}) \boldsymbol{v}+D(\boldsymbol{v}) \boldsymbol{v}+g(\boldsymbol{\Theta})=\boldsymbol{\tau}+\boldsymbol{w}
$$

The physical symbol mentioned above follows the standard specified by SNAME ${ }^{[22]}$. Where $\xi=[X, Y, Z]^{T} \in$ $R^{3}$ is the position vector of the vehicle in I-FRAME, $J(\Theta)$ is a transformation matrix which relates the BFRAME to the I-FRAME based on the Euler Angle $\boldsymbol{\Theta}=$ $[\theta, \phi, \psi]^{T}$, and $\boldsymbol{v}=[u, v, w, p, q, r]^{T} \in R^{6}$ is the set vector of velocity, according to the internationally accepted SNAME symbol definition, the six variables in this vector represent six velocity components of motion, namely surge, sway, heave, roll, pitch, and yaw respectively. $M=M_{R B}+$ $M_{A} \in R^{6 \times 6}$ is the inertia matrix of the vehicle. $C(\boldsymbol{v}) \in$ $R^{6 \times 6}$ and $D(\boldsymbol{v})=D+D_{2}(\boldsymbol{v}) \in R^{6 \times 6}$ represents Coriolis and centripetal matrix and damping matrix respectively, where $D$ is the linear damping and $D_{2}(\boldsymbol{v})$ is the twoorder damping. $\boldsymbol{\tau}=B_{c} \boldsymbol{u}$ is a 6-dimensional vector including the control forces and moments in B-FRAME, where $B_{c} \in R^{6 \times 4}$ is a control matrix and $\boldsymbol{u} \in R^{4}$ is a vector whose components are related to the thruster angular velocity in this paper. $\boldsymbol{w} \in R^{6}$ represents a disturbance vector acting on the vehicle.

The definition of the Cartesian coordinate system and motions are shown in Figure 4. More details about the above parameters can be found in reference ${ }^{[22]}$.

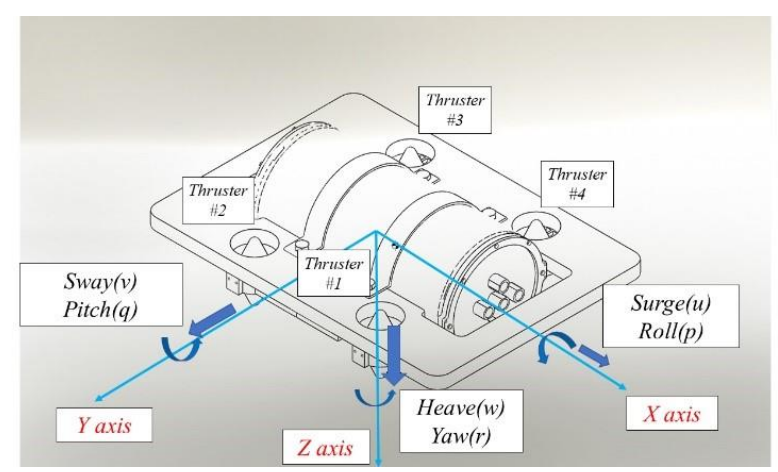

Figure 4 The Definition of ULSI-AUV's Coordinate System and Motion Mode

Because the configuration of thrusters of AUV developed in this paper is typically different from others, it is necessary to elaborate on control matrix $B_{c}$ and control vector $\boldsymbol{u}$. For the thrusters, the forces and moments are expressed as 


$$
\left[\begin{array}{l}
f_{z} \\
\tau_{x} \\
\tau_{y} \\
\tau_{z}
\end{array}\right]=\left[\begin{array}{cccc}
C_{t} & C_{t} & C_{t} & C_{t} \\
\frac{\sqrt{2}}{2} d C_{t} & -\frac{\sqrt{2}}{2} d C_{t} & -\frac{\sqrt{2}}{2} d C_{t} & \frac{\sqrt{2}}{2} d C_{t} \\
\frac{\sqrt{2}}{2} d C_{t} & \frac{\sqrt{2}}{2} d C_{t} & -\frac{\sqrt{2}}{2} d C_{t} & -\frac{\sqrt{2}}{2} d C_{t} \\
0 & 0 & 0 & 0
\end{array}\right]\left[\begin{array}{c}
\omega_{1}\left|\omega_{1}\right| \\
\omega_{2}\left|\omega_{2}\right| \\
\omega_{3}\left|\omega_{3}\right| \\
\omega_{4}\left|\omega_{4}\right|
\end{array}\right]
$$

Where the four items of the vector in the left equation represent the force along the $z$-axis and the torque around the $x$-axis, $y$-axis, and $z$-axis, respectively. $\omega_{i}(i=1,2,3,4)$ is the rotary speed of each thruster, which defined that if $\omega_{i}>0$ the thruster actuates in the clockwise direction, and vice versa. $d$ is the distance between the body centre of the vehicle and the thrusters. $C_{t}$ is integrated thrust coefficient, which satisfies $C_{t}=T / \omega^{2}$, where $T$ is the total force of one thruster.

With the expression above, the following can be obtained

$$
\begin{gathered}
\boldsymbol{u}=\left[u_{1}, u_{2}, u_{3}, u_{4}\right]^{T} \\
=\left[\omega_{1}\left|\omega_{1}\right|, \omega_{2}\left|\omega_{2}\right|, \omega_{3}\left|\omega_{3}\right|, \omega_{4}\left|\omega_{4}\right|\right]^{T} \\
B_{c}=\left[\begin{array}{cccc}
0 & 0 & 0 & 0 \\
0 & 0 & 0 & 0 \\
C_{t} & C_{t} & C_{t} & C_{t} \\
\frac{\sqrt{2}}{2} d C_{t} & -\frac{\sqrt{2}}{2} d C_{t} & -\frac{\sqrt{2}}{2} d C_{t} & \frac{\sqrt{2}}{2} d C_{t} \\
\frac{\sqrt{2}}{2} d C_{t} & \frac{\sqrt{2}}{2} d C_{t} & -\frac{\sqrt{2}}{2} d C_{t} & -\frac{\sqrt{2}}{2} d C_{t} \\
0 & 0 & 0 & 0
\end{array}\right]
\end{gathered}
$$

\section{Controller Design}

The proposed control system comprises outer-loop and inner-loop, which is shown in Figure 5. Due to the high nonlinearity of the system and the uncertainty of the hydrodynamic parameters, sliding mode control (SMC) is adopted to design the AUV's controller. Compared to other control methods like PID control, backstepping control and so on ${ }^{[23-24]}$, it has been successfully applied to the control of AUVs as shown in ${ }^{[25-27]}$ because of its robust capacity.

The control loop contains the whole position and attitude state vector

$$
\boldsymbol{x}=[\boldsymbol{v}, \xi]=[u, v, w, p, q, r, X, Y, Z, \theta, \phi, \psi]^{T}
$$

To design the control law better, we simultaneous equations (1)(2) to obtain the nonlinear state-space equations of the system, which can be written as

$$
\dot{\boldsymbol{x}}=A \boldsymbol{x}+B \boldsymbol{u}+f(\boldsymbol{x}, t)+d
$$

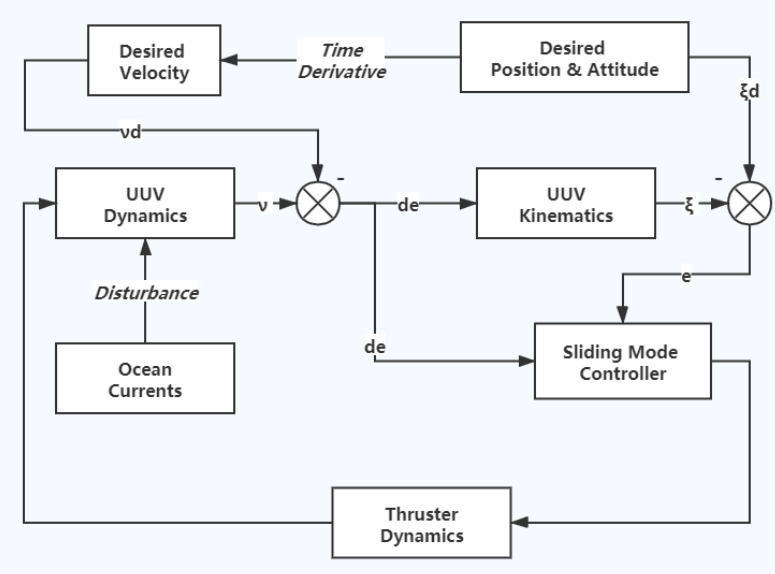

Figure 5 The position-speed closed-loop control diagram

It's easy to know that

$$
\begin{gathered}
A=\left[\begin{array}{c}
-M^{-1} D \\
0_{6 \times 6}
\end{array}\right], B=\left[\begin{array}{c}
-M^{-1} B_{c} \\
0_{6 \times 4}
\end{array}\right] \\
f(\boldsymbol{x}, t)=\left[\begin{array}{c}
-M^{-1}\left(C(\boldsymbol{v})+D_{2}(\boldsymbol{v})+g(\boldsymbol{q})\right) \\
J(\boldsymbol{q}) \boldsymbol{v}
\end{array}\right]
\end{gathered}
$$

Choose the following sliding mode surface:

$$
\begin{aligned}
& \sigma(x)=s^{T} x \\
& \dot{\sigma}(x)=s^{T} \dot{x}
\end{aligned}
$$

For some column vector $s \in R^{12}$ allows the use of pole-placement techniques in the design of the nonlinear controller.

To ensure the stability of the controller, a positive definite Lyapunov function has been considered as below

$$
V(\boldsymbol{x})=\frac{1}{2}[\sigma(\boldsymbol{x})]^{2}
$$

According to Barbalat lemma, we must guarantee that the sliding surface $\sigma$ will converge to zero in finite time by the condition

$$
\dot{\sigma}(\boldsymbol{x})=\left(\boldsymbol{s}^{T} \boldsymbol{d}-\boldsymbol{\eta}\right) \operatorname{sign}(\sigma(\boldsymbol{x}))
$$

The scalar $\eta>0$ is chosen too large enough to overcome the uncertainty of parameters and disturbances. 
Substituting Equation (11) into Equation (9) to get

$$
\dot{V}(\boldsymbol{x})=\sigma(\boldsymbol{x}) \dot{\sigma}(\boldsymbol{x})=\left(\boldsymbol{s}^{T} \boldsymbol{d}-\eta\right)|\sigma(\boldsymbol{x})| \leq 0
$$

Therefore, the global convergence of the system is guaranteed. Substituting Equation (7), (8), and (9) into Equation (11) to get

$$
\boldsymbol{s}^{T}(A \boldsymbol{x}+B \boldsymbol{u}+f(\boldsymbol{x}, t)+\boldsymbol{d})=\left(\boldsymbol{s}^{T} \boldsymbol{d}-\eta\right) \operatorname{sign}\left(\boldsymbol{s}^{T} \boldsymbol{x}\right)
$$

We can deduce that the ideal controller is

$$
\boldsymbol{u}=-B_{s}^{+}\left(\boldsymbol{s}^{T} A \boldsymbol{x}+\boldsymbol{s}^{T} f(\boldsymbol{x}, t)+\eta \operatorname{sign}\left(\boldsymbol{s}^{T} \boldsymbol{x}\right)\right)
$$

Where $B_{S}^{+}$is the pseudo-inverse matrix of $\boldsymbol{s}^{T} B$. It's important to emphasize that $\boldsymbol{u}$ may have multiple solutions because $\boldsymbol{s}^{T} B$ is not a square matrix.

\section{Experimental and Numerical Results}

The above ULSI-AUV mathematical model and sliding mode controller model is established and simulated in MATLAB/Simulink, the random ocean currents and error of measurement are both taking into account. The desired heave velocity, depth, roll angle and pitch angle are set as $0.06 \mathrm{~m} / \mathrm{s}, 3 \mathrm{~m}, 0 \mathrm{deg}$, $0 \mathrm{deg}$ respectively. Set the ULSI-AUV to fix its depth for 250 s after submerging to the specified depth, and then float up automatically. Because of the forces produced by four thrusters having value along the $z$-axis, it should be noted that the surge, sway and yaw motion cannot be achieved.

Experimental tests are carried out in the engineering basin in the Ship Model Towing Pool Laboratory in Huazhong University of Science and Technology. The engineering basin is $100 \mathrm{~m}$ long, $2 \mathrm{~m}$ wide and $5 \mathrm{~m}$ depth. Figure 6 shows the station keeping control test of ULSIAUV prototype in the basin.

The heave depth information was read by the depth sensor namely B30-MS5837 which can measure up to $300 \mathrm{~m}$ depth with a depth resolution of $10 \mathrm{~mm}$, and the roll and pitch information was obtained from the onboard 6-DOF attitude sensor which dynamic accuracy is within 0.1 degrees.

The effective PWM duty ratio of bi-directional electronic tuning is between 0.05 and 0.1 (the corresponding PWM value is $1000 \sim 2000$ in this experiment), and 0.75 (PWM value is 1500 ) is the median. Considering the requirement of a weak current, the PWM duty ratio is set to be between 0.7 and 0.8 (PWM value is 1400 1600), and the total current can be observed from the panel of the DC regulated power supply in real-time during the experiment, which is always less than $500 \mathrm{~mA}$.

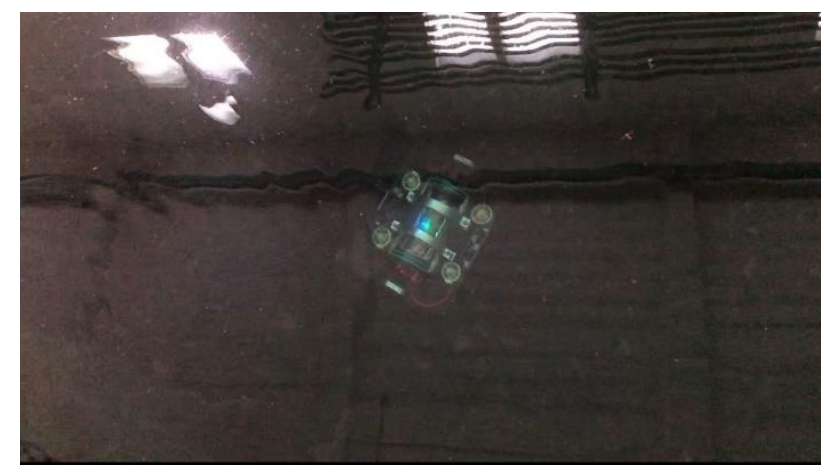

Figure 6 Station keeping control test in the basin

Since low-cost gyroscopes and depth sensors are used, the frequency of the MCU input signal is only about $10 \mathrm{~Hz}$, and the frequency of data transmission to the host computer is also less than $10 \mathrm{~Hz}$. The upper computer software is programmed based on the MATLAB GUI, as shown in Figure 7. The initial state, target speed, depth, angle, working time and other parameters of the experiment are the same as the corresponding parameters of the simulation. The purpose of the experiment is to compare with the simulation results and validate the feasibility of the control method to adopt the control law in future projects.

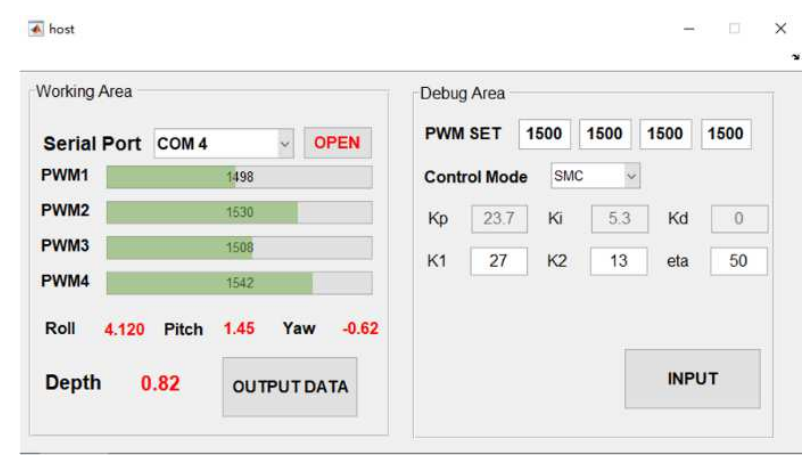

Figure 7 Upper computer software user interface

Related numerical and experimental results are shown in Figure 8-10. Figure 8 shows the comparison of experimental results, numerical results and the desired value of depth. It can be seen that the actual time to reach the predetermined depth is slightly longer than the simulation time by about 2 seconds, which may be due to the following reasons: Firstly, there is a time delay when the thruster is started; secondly, due to motor ageing or other reasons, under the same PWM duty ratio, the actual output thrust is slightly smaller than the ideal value and the speed 
is slightly slower. However, the experimental and simulation results are not much different from the expected results. Especially after reaching steady state, the depth error collected from experi-mental data is not more than 20 $\mathrm{mm}$. Considering the accuracy of the depth sensor is already $10 \mathrm{~mm}$, it can be concluded that the control law is robust to depth control and the process is reliable.

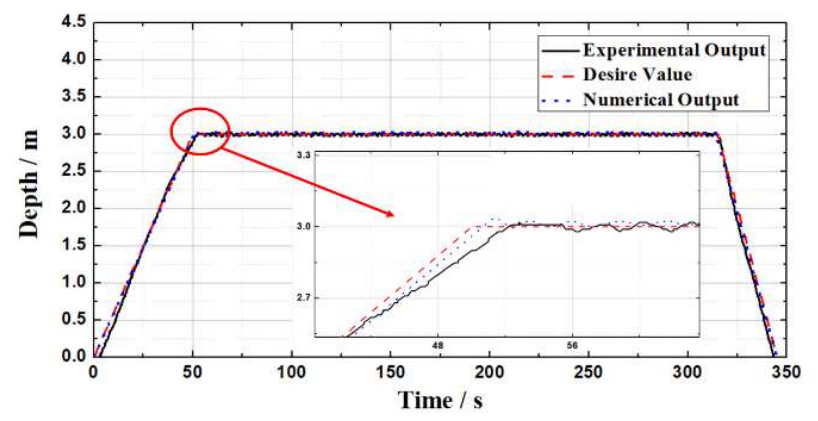

Figure 8 Depth output and desire value

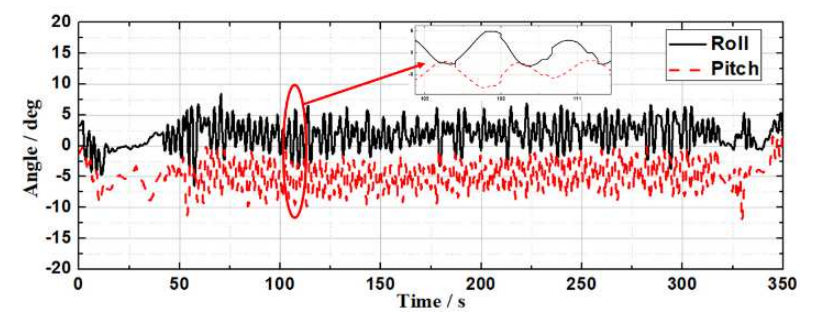

Figure 9 Experimental result of roll and pitch angle

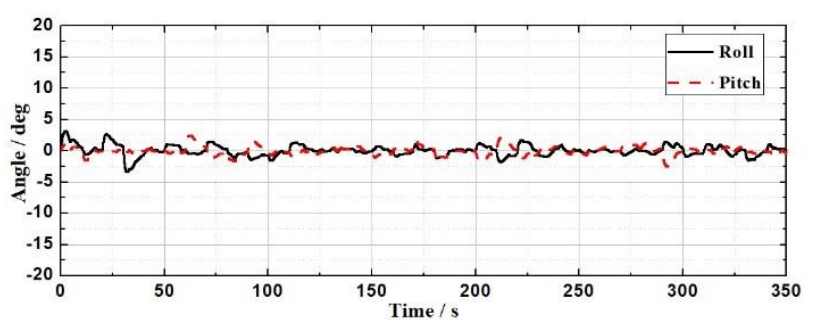

Figure 10 Numerical result of roll and pitch angle

Figures 9-10 show the numerical simulation results and experimental results of roll and pitch angles respectively. Because the wave disturbance is added in the numerical simulation process, the attitude angle always has an oscillation near the median, and the amplitude is less than 5 degrees, which is ideal. However, there is a steady-state error of about -5 degrees in the experimental results of pitch angle, which may be due to the following reasons: Firstly, there are still slight errors between the centre of gravity and the centre of buoyancy in the $x$-direction and the $y$-direction, resulting in a negative steady-state angle. Secondly, the CFD results ignore higher-order terms, resulting in inaccurate model parameters. Thirdly, the non-zero buoyancy cable is connected to the tail of ULSI-AUV, which causes great interference to pitch angle. To best of our knowledge, the third reason may be the core of the problem. However, as can be seen in the enlarged view of Figure 9, the oscillation frequency of ULSI-AUV is about $0.5-1 \mathrm{~Hz}$, which will not cause interference to the system, and the oscillation amplitude is always limited within 10 degrees, which will not cause interference to the depth sensor.

\section{Conclusion}

In this paper, a dynamic station keeping method has been proposed for ULSI-AUV control. Based on the result, the sliding mode controller has been designed for fixed-depth and anti-rolling. Numerical analysis has been provided to show the effectiveness of the proposed algorithm. An experimental result has also been provided to validate the presented method. Further research on the development of this prototype will focus on the effects of uncertainty in the model parameter calculations and improve the hardware like increasing data transmission frequency.

\section{Declaration}

\section{Acknowledgements}

Sincerely thanks to Mr Zhen-Zhen Zhao, Mr Biao Liu, Mr Yuan Li for the cooperation in the project.

\section{Funding}

Supported by National Science and Technology. Major Project: 2016ZX05038-004-LH001.

\section{Availability of data and materials}

The main design parameters supporting the conclusions of this article are included within the article. The material of each mechanical component are shown in Table 2.

Table 2 The material of each mechanical component

\begin{tabular}{cc}
\hline Component & Material \\
\hline Watertight enclosure & Polymethyl methacrylate \\
End cover & Polymethyl methacrylate \\
Flange & Aluminium alloy \\
Threading screw & Aluminium alloy \\
Snap ring & Stainless steel \\
Counterbalance component & Lead \\
\hline
\end{tabular}

Authors' contributions 
The author' contributions are as follows: Huan Zheng was in charge of the whole trial; Yu-Hao Zeng wrote the manuscript; $\mathrm{Zu}-\mathrm{Yao} \mathrm{Yu}$ assisted with sampling and laboratory analyses.

\section{Competing interests}

The authors declare no competing financial interests.

\section{Reference}

[1] Chutia S, Kakoty N M, Deka D. A review of underwater robotics, navigation, sensing techniques and applications[M]//Proceedings of the Advances in Robotics. 2017: $1-6$.

[2] Liu G, Yuan Z, Qiu Z, et al. A brief review of bio-inspired surface technology and application toward underwater drag reduction[J]. Ocean Engineering, 2020, 199: 106962.

[3] Stojanovic M, Preisig J. Underwater acoustic communication channels: Propagation models and statistical characterization[J]. IEEE communications magazine, 2009, 47(1): 84-89.

[4] Domingo M C. Overview of channel models for underwater wireless communication networks $[\mathrm{J}]$. Physical Communication, 2008, 1(3): 163-182.

[5] Stojanovic M. Underwater wireless communications: Current achievements and research challenges[J]. IEEE Oceanic Engineering Society Newsletter, 2006, 41(2): 1-5.

[6] Shaneyfelt T, Joordens $M$ A, Nagothu $K$, et al. RF communication between surface and underwater robotic swarms[C]//2008 World Automation Congress. IEEE, 2008: 1-6.

[7] Zhu Q, Xiong W, Liu H, et al. A brief review of underwater electric current communication[C]//2016 IEEE 20th International Conference on Computer Supported Cooperative Work in Design (CSCWD). IEEE, 2016: 465-469.

[8] Li J H, Kim M G, Kang H J, et al. Development of UUV platform and its control method to overcome strong currents: simulation and experimental studies[J]. IFAC-PapersOnLine, 2018, 51(29): 268-273.

[9] Santhakumar M, Asokan T. Power efficient dynamic station keeping control of a flat-fish type autonomous underwater vehicle through design modifications of thruster configuration[J]. Ocean Engineering, 2013, 58: 11-21.

[10] Koh T H, Lau M W S, Seet G, et al. A control module scheme for an underactuated underwater robotic vehicle $[\mathrm{J}]$. Journal of Intelligent and Robotic Systems, 2006, 46(1): 43-58.

[11] Serrani A. Robust station-keeping of underwater vehicles under velocity measurement offsets[J]. IFAC Proceedings Volumes, 2004, 37(10): 333-338.

[12] Lots J F, Lane D M, Trucco E, et al. A 2D visual servoing for underwater vehicle station keeping[C]//Proceedings 2001 ICRA. IEEE International Conference on Robotics and Automation (Cat. No. 01CH37164). IEEE, 2001, 3: 2767-2772.

[13] Santhakumar M, Asokan T. Power efficient dynamic station keeping control of a flat-fish type autonomous underwater vehicle through design modifications of thruster configuration[J]. Ocean Engineering, 2013, 58: 11-21.

[14] Crouse G. Conceptual design of a submersible airplane[C]//48th AIAA Aerospace Sciences Meeting Including the New Horizons Forum and Aerospace Exposition. 2010: 1012.

[15] Drews P L J, Neto A A, Campos M F M. Hybrid unmanned aerial underwater vehicle: Modeling and simulation[C]//2014 IEEE/RSJ International Conference on Intelligent Robots and Systems. IEEE, 2014: 4637-4642.

[16] Bian J, Xiang J. QUUV: A quadrotor-like unmanned underwater vehicle with thrusts configured as X shape[J]. Applied Ocean Research, 2018, 78: 201-211.

[17] Suarez Fernandez R A, Parra R, Andres E, et al. Nonlinear Attitude Control of a Spherical Underwater Vehicle[J]. Sensors, 2019, 19(6): 1445.

[18] Allotta B, Baines S, Bartolini F, et al. Design of a modular Autonomous Underwater Vehicle for archaeological investigations[C]//OCEANS 2015-Genova. IEEE, 2015: 1-5.

[19] Allen B, Stokey R, Austin T, et al. REMUS: a small, low cost AUV; system description, field trials and performance results[C]//Oceans' 97. MTS/IEEE Conference Proceedings. IEEE, 1997, 2: 994-1000.

[20] Choi H T, Hanai A, Choi S K, et al. Development of an underwater robot, ODIN-III[C]//Proceedings 2003 IEEE/RSJ International Conference on Intelligent Robots and Systems (IROS 2003)(Cat. No. 03CH37453). IEEE, 2003, 1: 836-841.

[21] Fossen T I. Guidance and control of ocean vehicles[M]. New York: Wiley, 1994.

[22] SNAME T. Nomenclature for treating the motion of a submerged body through a fluid[J]. The Society of Naval Architects and Marine Engineers, Technical and Research Bulletin No, 1950: 1-5.

[23] Serrani A. Robust station-keeping of underwater vehicles under velocity measurement offsets[J]. IFAC Proceedings Volumes, 2004, 37(10): 333-338.

[24] Ansari U, Bajodah A H, Alam S. Generalized dynamic inversion based attitude control of autonomous underwater vehicles[J]. IFAC-PapersOnLine, 2016, 49(23): 582-589.

[25] Zhang G, Huang H, Wan L, et al. A novel adaptive second order sliding mode path following control for a portable AUV[J]. Ocean Engineering, 2018, 151: 82-92.

[26] Cristi R, Papoulias F A, Healey A J. Adaptive sliding mode control of autonomous underwater vehicles in the dive plane $[\mathrm{J}]$. IEEE journal of Oceanic Engineering, 1990, 15(3): 152-160.

[27] Healey A J, Lienard D. Multivariable sliding mode control for autonomous diving and steering of unmanned underwater vehicles[J]. IEEE journal of Oceanic Engineering, 1993, 18(3): 327-3. 
Figures

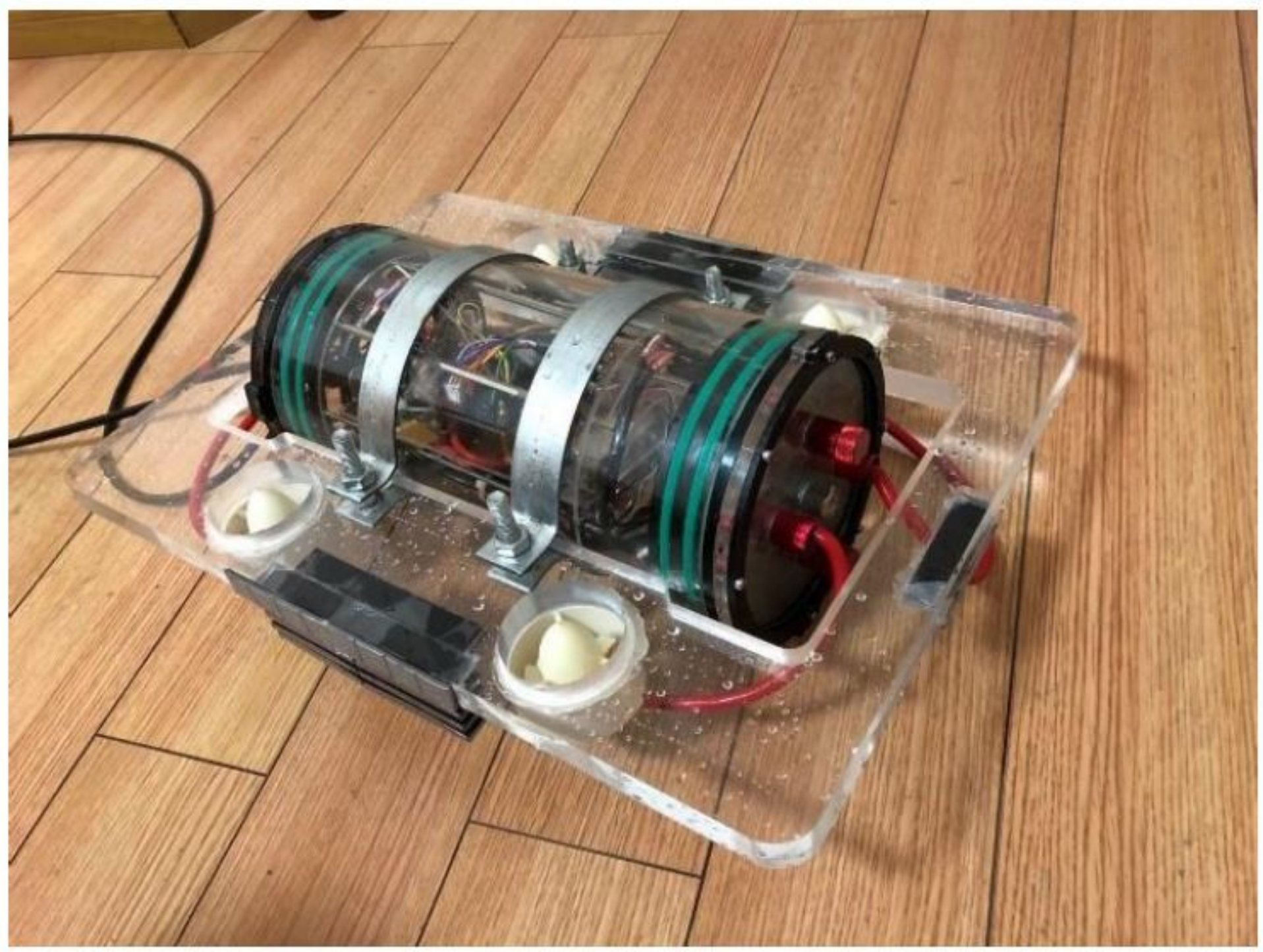

Figure 1

The ULSI-AUV Prototype 


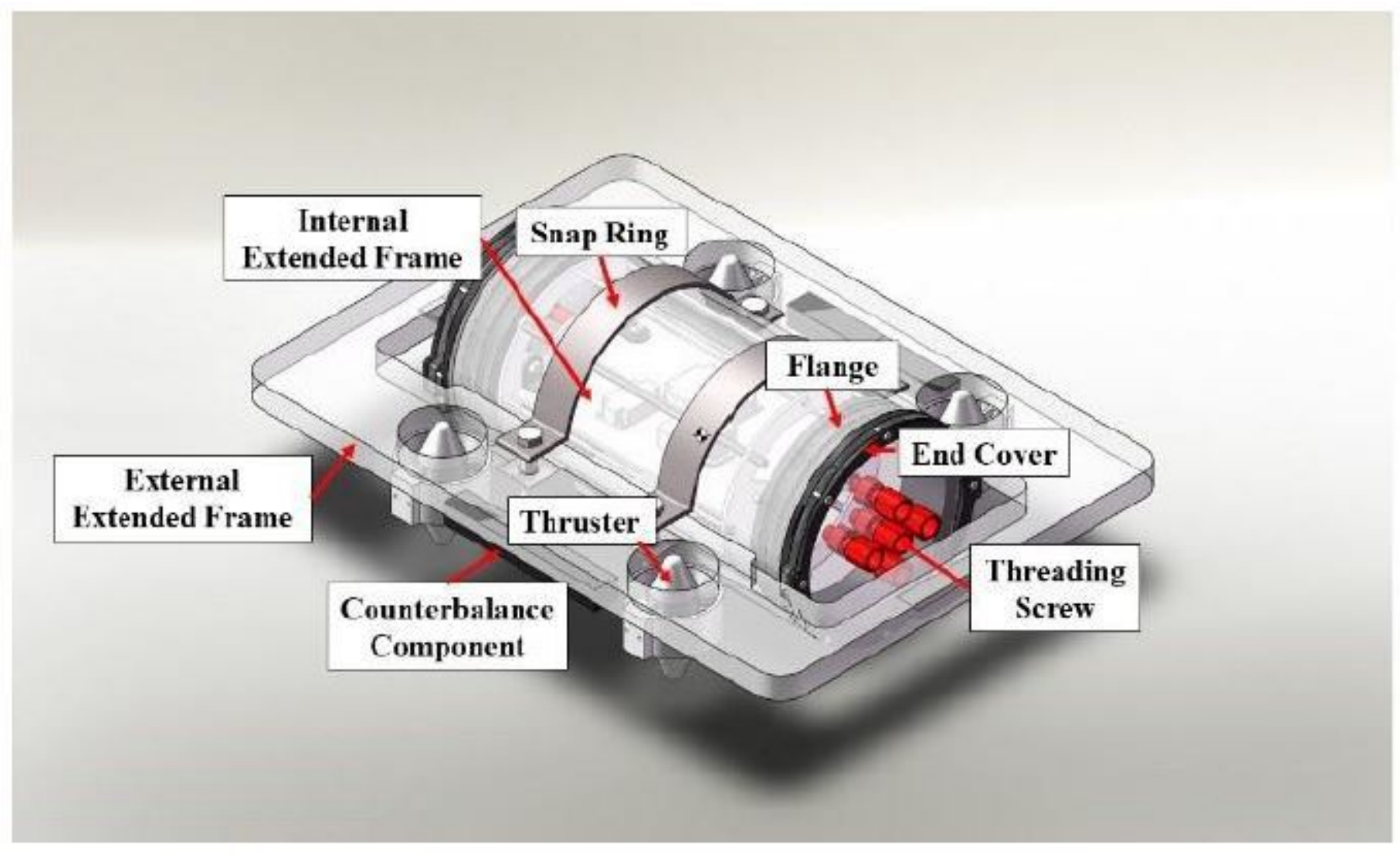

Figure 2

The ULSI-AUV 3D Model

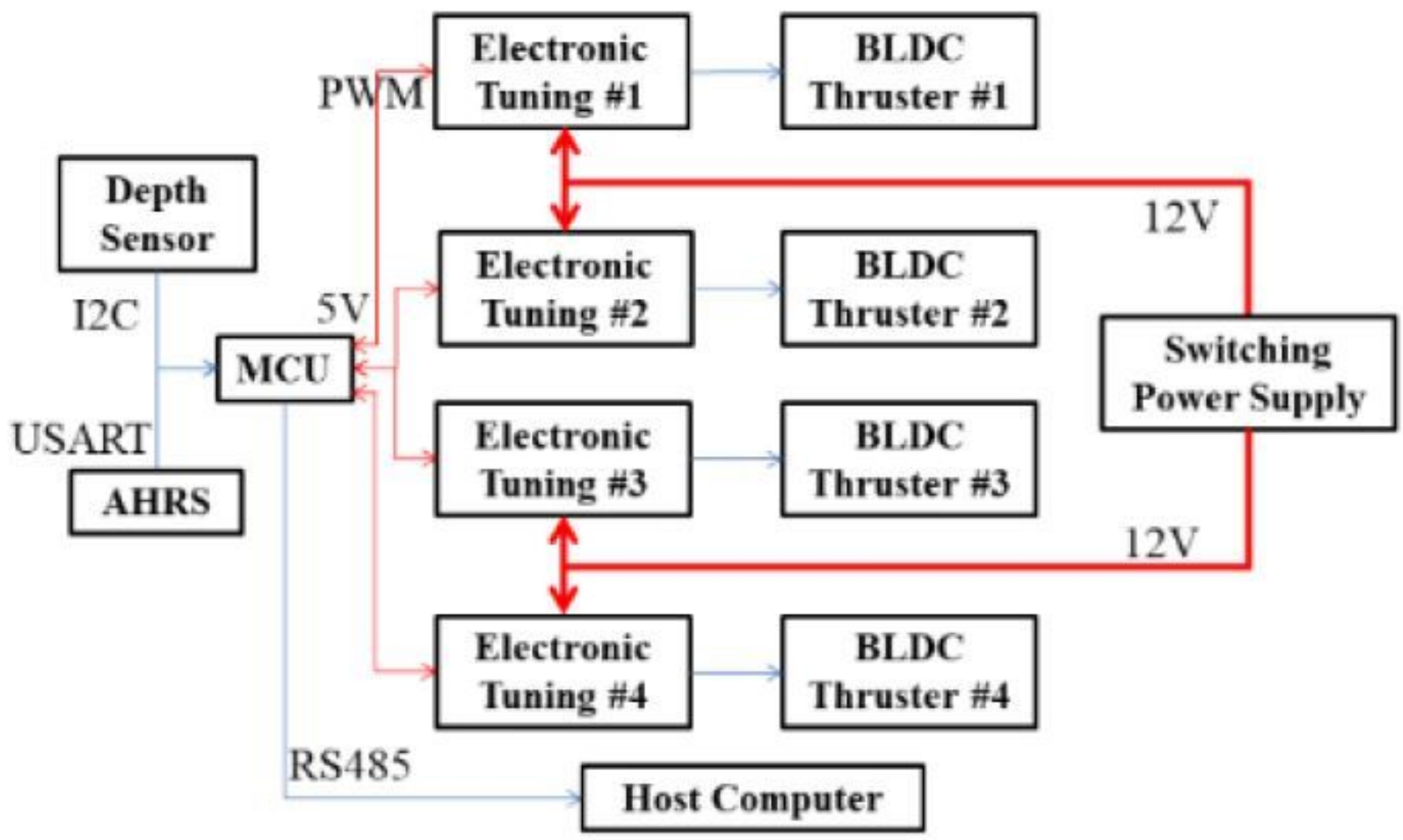


Figure 3

The ULSI-AUV's Electrical System

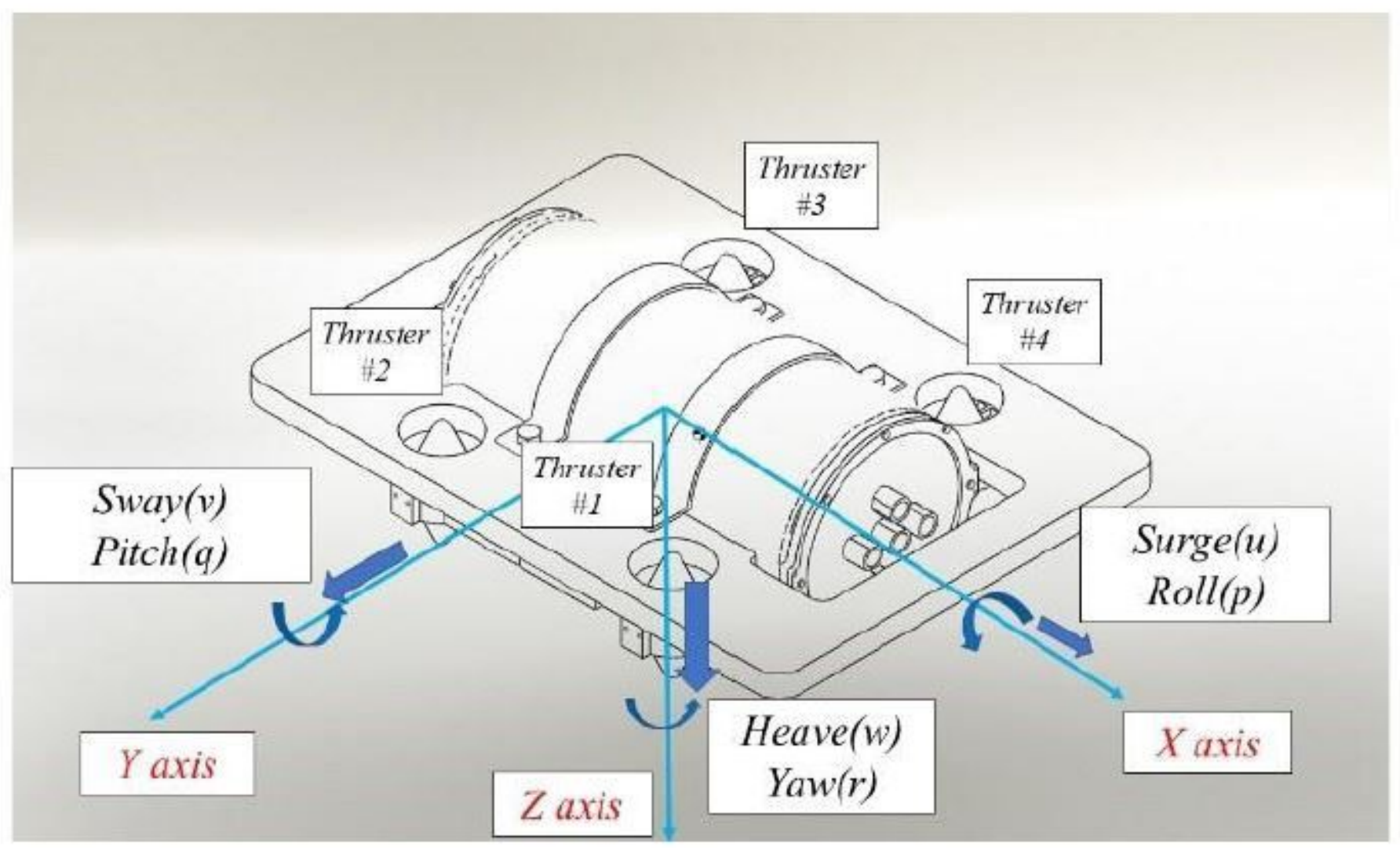

Figure 4

The Definition of ULSI-AUV's Coordinate System and Motion Mode 


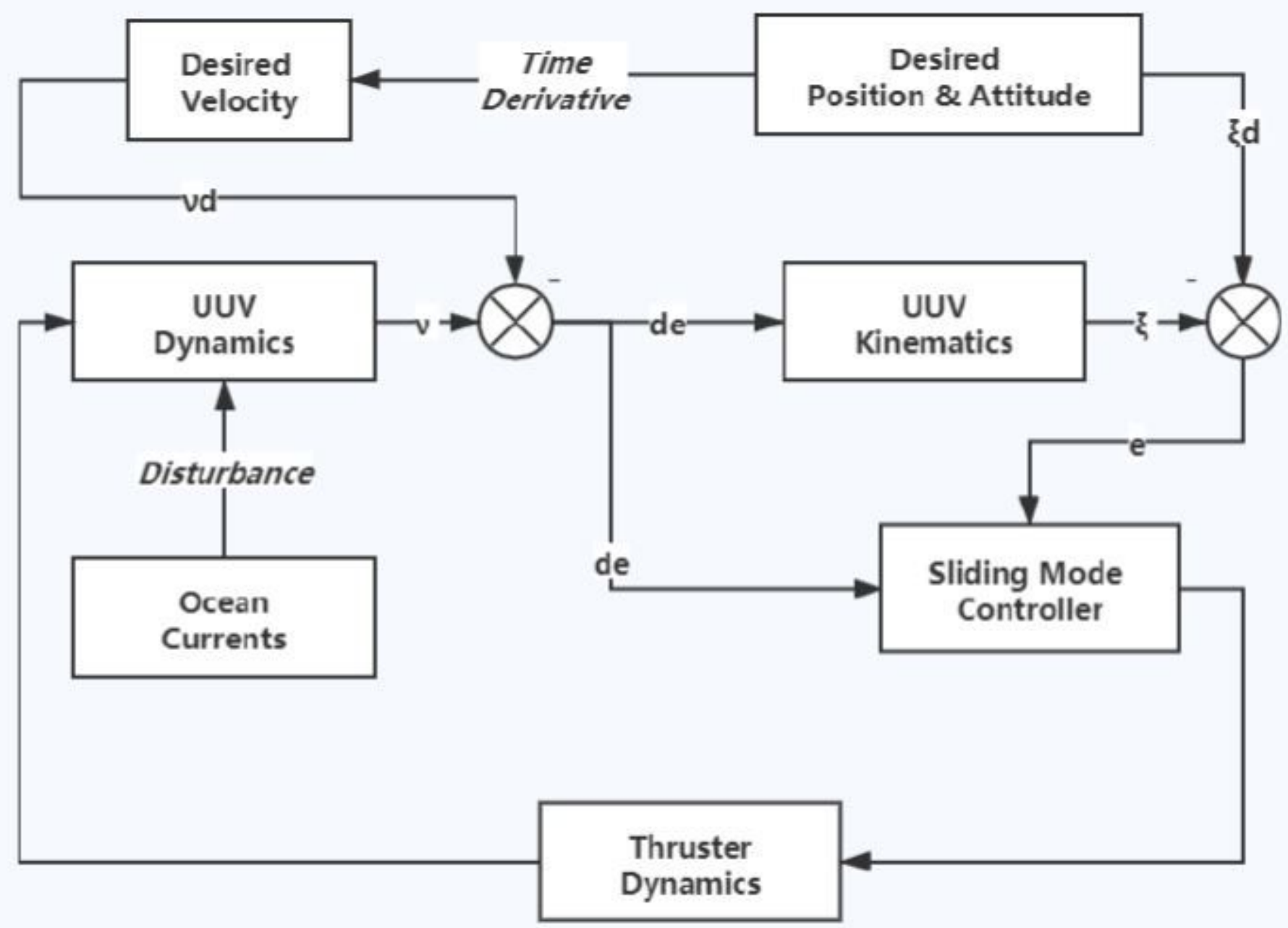

Figure 5

The position-speed closed-loop control diagram 


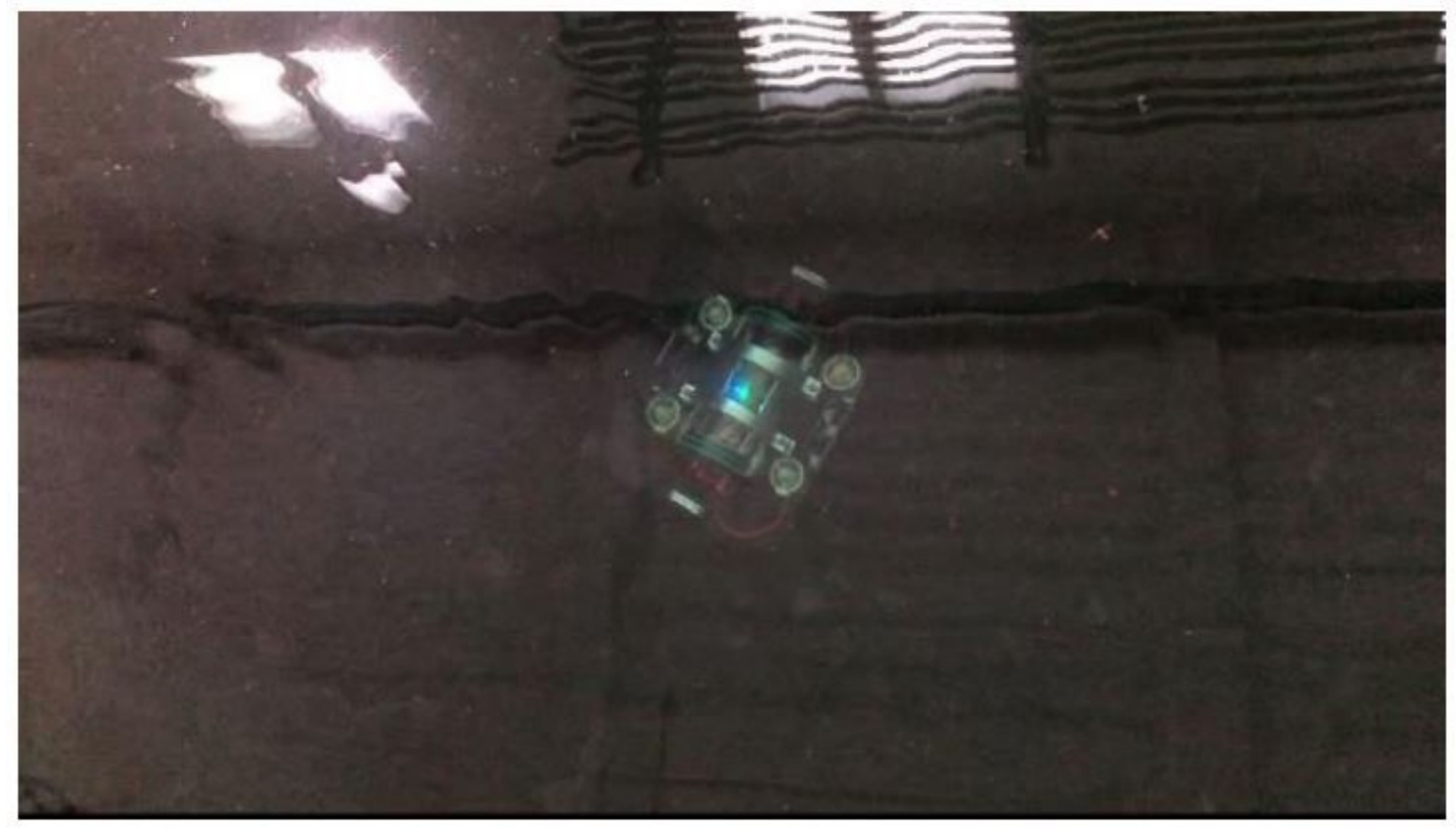

Figure 6

Station keeping control test in the basin

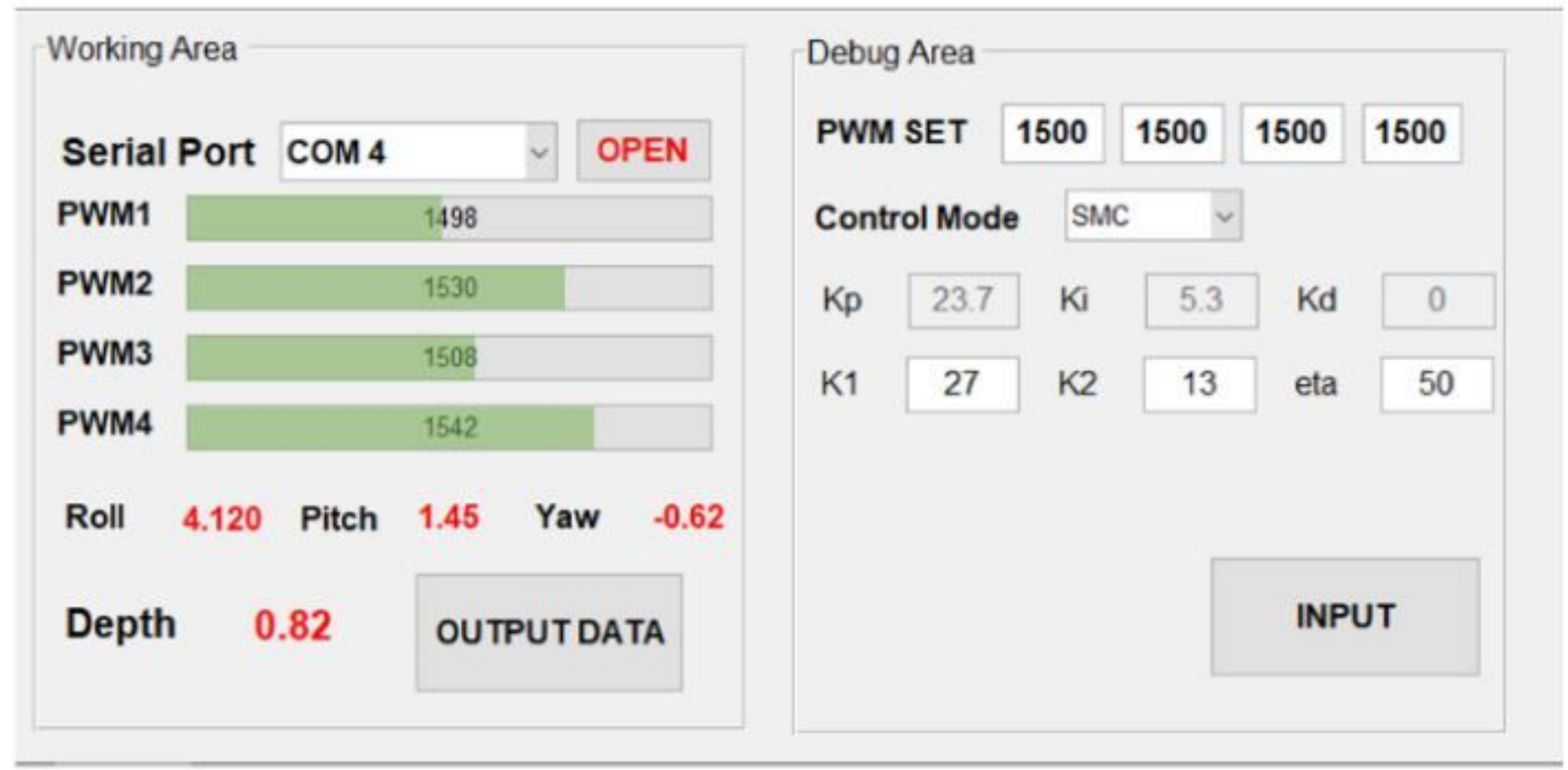

Figure 7

Upper computer software user interface 


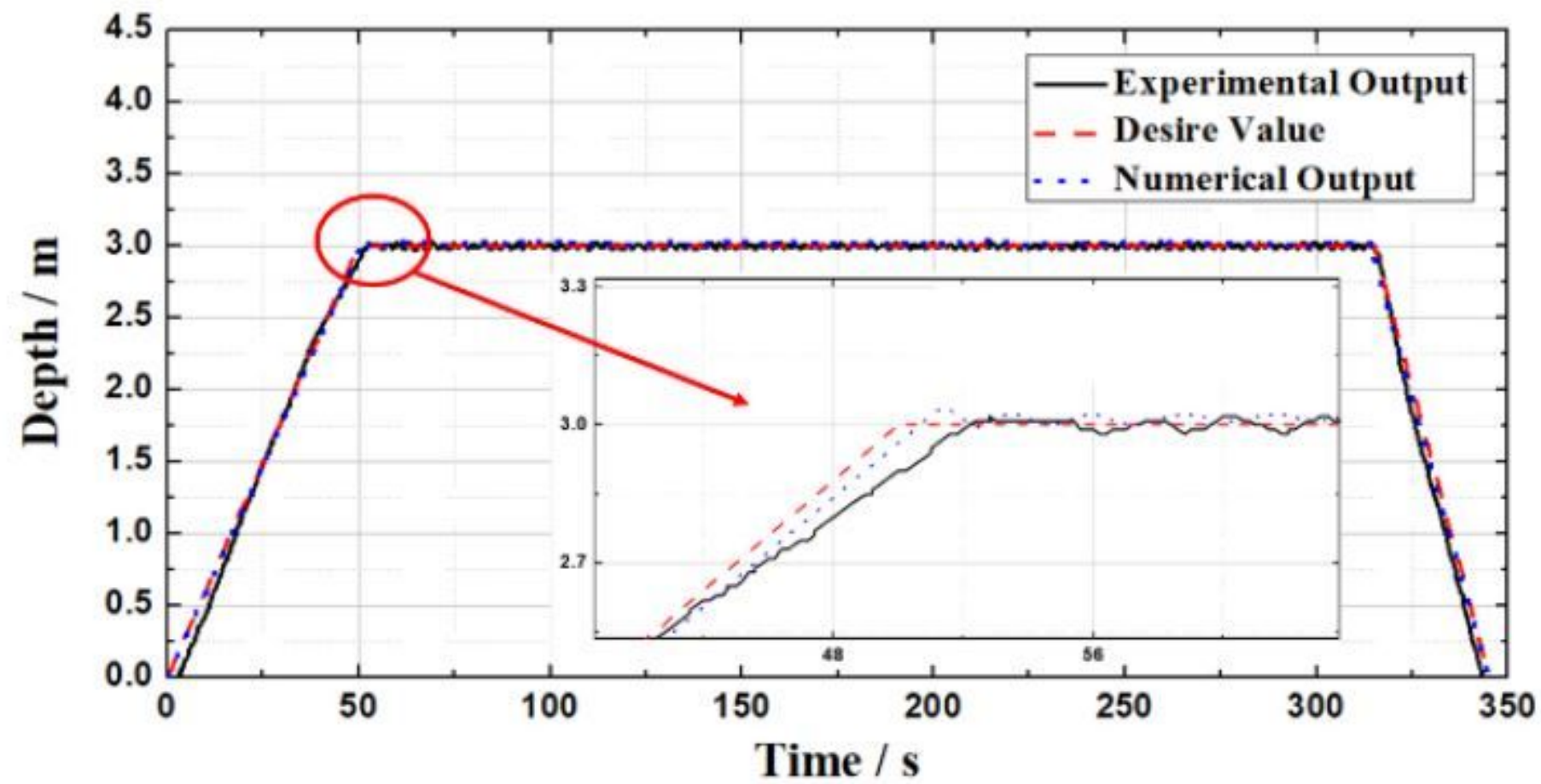

Figure 8

Depth output and desire value

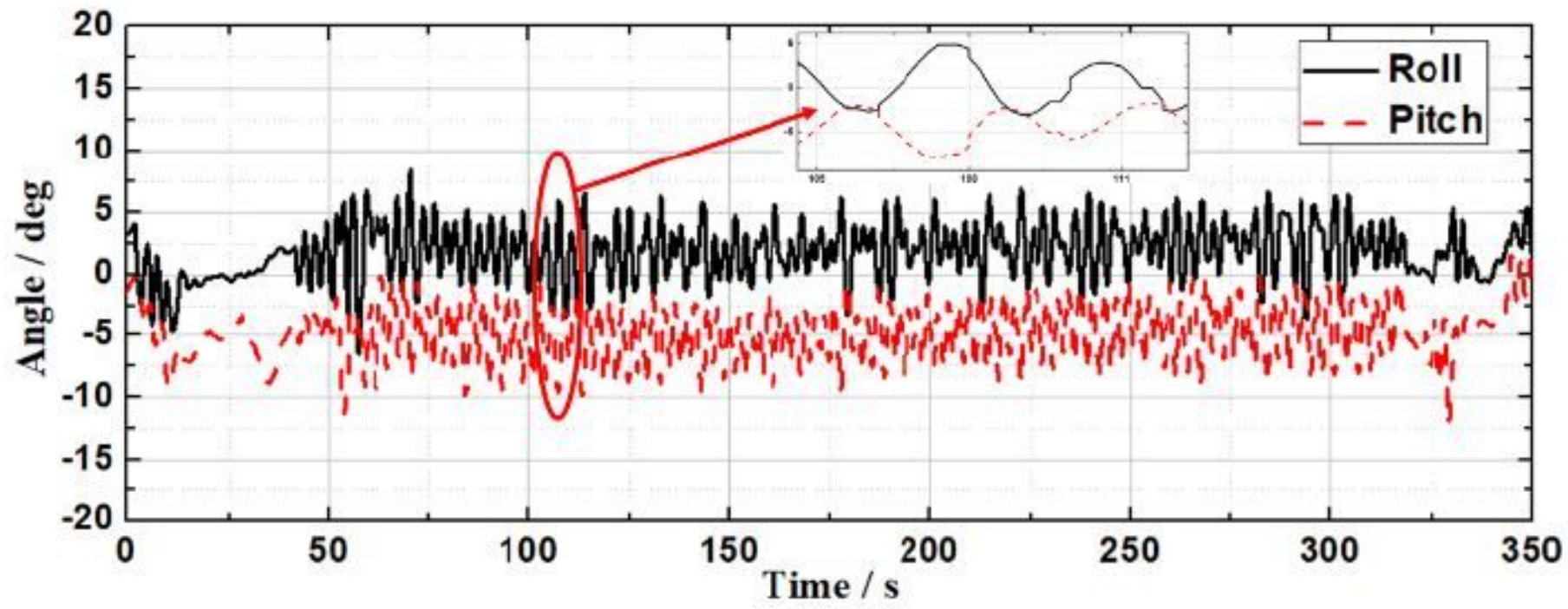

Figure 9

Experimental result of roll and pitch angle 


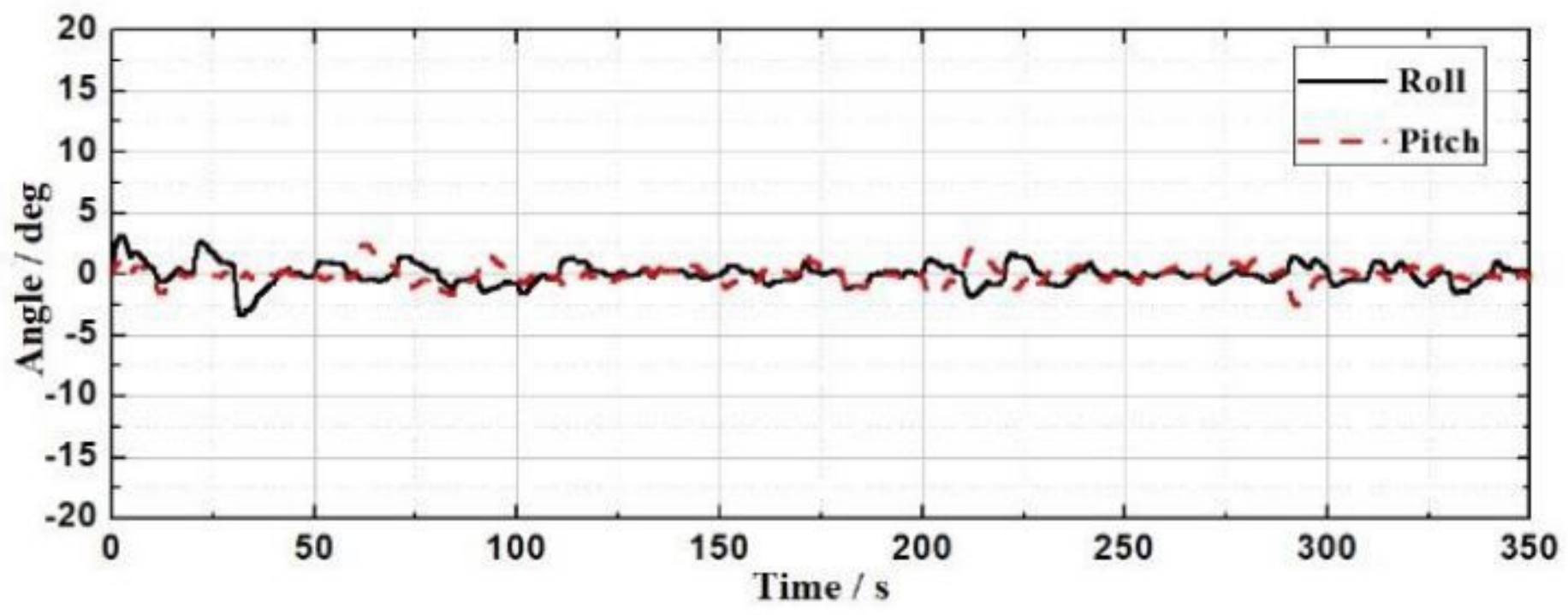

Figure 10

Numerical result of roll and pitch angle 